\title{
Photodecarbonylation of Diphenylcyclopropenone - a Direct Pathway to Electronically Excited Diphenylacetylene?
}

\author{
By Hendrik Vennekate ${ }^{1,2}$, Arne Walter ${ }^{1,2}$, Daniel Fischer ${ }^{1,2}$, Jörg Schroeder ${ }^{1,2, *}$, and \\ Dirk Schwarzer ${ }^{1,2}$ \\ 1 Max-Planck-Institute of Biophysical Chemistry, Am Faßberg 11, 37077 Göttingen, Germany \\ 2 Institute of Physical Chemistry, Georg-August-University Göttingen, Tammannstr. 6, 37077 Göttingen, \\ Germany
}

Dedicated to Prof. Horst Hippler on the occasion of his $65^{\text {th }}$ birthday

(Received August 11, 2011; accepted in revised form August 16, 2011)

\section{Reaction Dynamics / Photochemistry / Femtosecond IR-Spectroscopy}

A comparison of combined IR and UV/Vis pump-probe measurements of diphenylcyclopropenone (DPCP) and diphenylacetylene (DPA) provides clear evidence that the photodecarbonylation of DPCP following excitation to its $S_{2}$-state proceeds non-adiabatically to the electronic ground state of DPA. It is shown that transient absorption of electronically excited DPA is caused exclusively by photoexcitation of ground state DPA generated by preceding DPCP photodecarbonylation.

\section{Introduction}

The photochemistry of diphenylcyclopropenone (DPCP) is dominated by decarbonylation and the formation of diphenylacetylene (DPA) [1]. For $337 \mathrm{~nm}$ excitation, e.g., the quantum yield of this reaction is reported to be $1.00 \pm 0.03$ in benzene [2]. While thermodynamic quantities such as reaction enthalpies and reaction volumes have been studied in detail [3-5], the dynamics of this process has been investigated less extensively. In particular the question whether the reaction is adiabatic, proceeding on an excited state surface to form electronically excited DPA, or rather non-adiabatic, leading to ground state DPA, is discussed controversially. Basically three hypotheses concerning the decarbonylation path from the initially excited $S_{1}$-state of DPCP to DPA have been put forward. They are depicted schematically in Fig. 1.

Path I. From first picosecond transient absorption measurements, Hirata and Mataga concluded that the photodissociation of DPCP in solution was much faster than their

\footnotetext{
* Corresponding author. E-mail: jschroe2@gwdg.de
} 


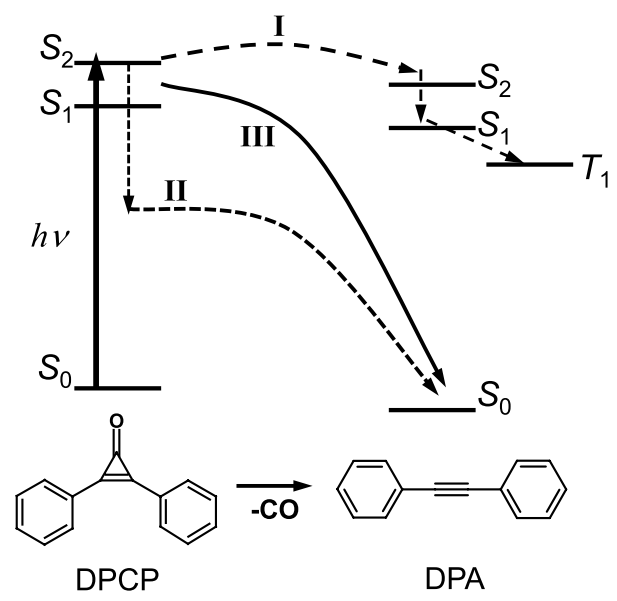

Fig. 1. Reaction pathways for the photodecarbonylation of diphenylcyclopropenone (DPCP).

experimental time resolution of several picoseconds and what they observed as an immediate product was DPA in the $S_{2}$ state [6]. This interpretation was based on the similarity of the transient spectra and kinetics with those of photo-excited DPA and later confirmed by femtosecond transient absorption experiments [7]. In cyclohexane solution, a lifetime of $200 \mathrm{fs}$ was measured for the DPCP $S_{2}$-state which was supposed to decay directly to the $S_{2}$-state of DPA. The subsequent photophysics of DPA was characterized by time constants of $8 \mathrm{ps}$ for $S_{2} \rightarrow S_{1}$ internal conversion and $210 \mathrm{ps}$ for $S_{1} \rightarrow T_{1}$ intersystem crossing. Recently, this adiabatic photodecarbonylation path on the excited singlet surface was suggested to be responsible for a hypothetical quantum chain mechanism in solid crystalline DPCP consisting of alternating steps of adiabatic formation of $\operatorname{DPA}\left(S_{2}\right)$ and electronic energy transfer from $\operatorname{DPA}\left(S_{2}\right)$ to $\operatorname{DPCP}\left(S_{0}\right)$ [8]. The mechanism was proposed to explain the about threefold enhancement of the photodissociation quantum yield of DPCP observed in the crystalline solid phase with respect to liquid solution.

Path II. Quantum chemical calculations based on time-dependent DFT and CIS methods led to the suggestion that the decomposition takes place from the vibrationally hot DPCP ground state [9]. This mechanism would require fast $S_{2} \rightarrow S_{0}$ internal conversion within DPCP prior to dissociation.

Path III. Femtosecond pump-probe experiments on DPCP derivatives and naphthyl substituted cyclopropenones led Poloukhtine and Popik to the conclusion that cyclopropenone decarbonylation in general takes place via a short-lived intermediate on the electronically excited singlet surface from where the reaction proceeds to ground state acetylene [10]. On the basis of supplementary nanosecond laser flash photolysis measurements, the authors suggested that signals of electronically excited acetylenes arise from product accumulation due to photo-fragmentation of the cyclopropenones. In case of the DPCP-derivative di-( $p$-methoxyphenyl)cyclopropenone, electronic spec- 
tral overlap with the acetylene product is less severe than for DPCP and formation and decay time constants of the postulated intermediate in methanol were on the order of $0.5 \mathrm{ps}$. The authors also mention the observation of complex spectral dynamics in the case of DPCP, which they apparently interpret in terms of the sequence $\operatorname{DPCP}\left(S_{2}\right) \rightarrow \operatorname{DPCP}\left(S_{1}\right) \rightarrow \operatorname{DPA}\left(S_{1}\right) \rightarrow \operatorname{DPA}\left(S_{0}\right)$ without providing any time constants. It is not clear, why they thought the lowest excited singlet states of DPCP and DPA were involved instead of the intermediate postulated for the other compounds.

The aim of this work is to establish the DPCP decarbonylation pathway by employing femtosecond infrared (IR) and broadband continuum probe spectroscopy to study the DPCP photoreaction using excitation wavelengths in the range 267-340 nm and thereby covering the first and second absorption band of DPCP. As vibrational absorption bands are narrower than electronic spectra, the assignment of intermediates is facilitated. Furthermore, absolute quantum yields of primary reaction steps can easily be determined by comparing intensities of photo-bleached and -generated vibrational marker bands. Applying femtosecond IR spectroscopy, we recently elucidated photodecomposition mechanisms of several organic peroxides in this way [11-13].

\section{Experimental}

The experiments were performed with a laser system based on a $1 \mathrm{kHz}$ Ti:sapphire oscillator/regenerative amplifier producing $150 \mathrm{fs}$ pulses at $800 \mathrm{~nm}$ with pulse energies of $0.7 \mathrm{~mJ}$. The output energy was split to independently pump two optical parametric amplifiers [14] (OPA). One OPA was used to generate pump pulses in the wavelength range $295-340 \mathrm{~nm}$ by fourth harmonic generation of the signal wave. Alternatively, a pump wavelength of $267 \mathrm{~nm}$ was produced by third harmonic generation of a small portion of the $800 \mathrm{~nm}$ regenerative amplifier output. Pump pulse energies entering the sample were $1-2 \mu \mathrm{J}$. Tunable mid-infrared probe pulses were generated by difference frequency mixing of signal and idler pulses from the second OPA. The IR pulses were split into a probe and a reference beam. The former was superimposed on the pump beam and both were focused into the sample cell. The relative plane of polarization was set to $54.7^{\circ}$. Behind the sample, reference and probe beam were directed to a polychromator and their spectra were imaged onto a liquid nitrogen cooled $\mathrm{HgCdTe}$ detector of $2 \times 32$ pixels with $11.4 \mathrm{~nm}$ resolution. To avoid spectral and temporal distortion of the IR pulses by $\mathrm{CO}_{2}$ and water absorptions in air, the entire pump-probe setup was purged with dry nitrogen. Stationary IR-spectra of DPCP and DPA were taken using an FT-IR spectrometer (Spectrum BX, Perkin Elmer) with $0.5 \mathrm{~cm}^{-1}$ resolution.

In addition, transient spectra in the near UV and visible were measured with a white-light continuum probe pulse. The continuum was generated by focusing $10 \mu \mathrm{J}$ of the amplified $800 \mathrm{~nm}$ pulse into a $\mathrm{CaF}_{2}$ crystal of $4 \mathrm{~mm}$ length and split into a reference and probe pulse. The latter was overlaid with the UV pump pulse at the sample. Each continuum was detected with a 256 element linear diode array attached to a spectrograph. The measured transient spectra were corrected with respect to the group delay dispersion of the probe continuum.

The experiments with $10 \mathrm{mM}$ solutions of DPCP and DPA in acetonitrile were performed in a stainless steel flow cell with $1.0 \mathrm{~mm} \mathrm{CaF}_{2}$ windows. The optical path length 


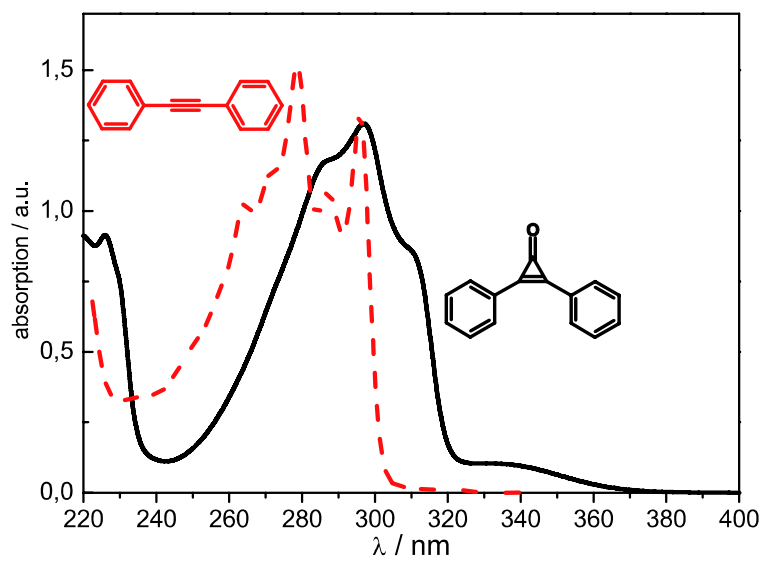

Fig. 2. UV absorption spectra of DPCP (solid) and DPA (dashed) in acetonitrile.

inside the cell was $0.6 \mathrm{~mm}$. The flow rate was adjusted to assure replacement of the sample solution between successive pump laser pulses.

DPCP (98\%) was purchased from Aldrich and purified by column chromatography (silica gel, solvent: ethyl acetate). After evaporating the solvent DPCP turned out to be contaminated with $0.2 \%$ of DPA as verified by HPLC chromatography. Recrystallisation from petrol ether 60/80 reduced the DPA impurity to $<0.05 \%$. DPA $(>99 \%$ ) was purchased from ABCR and used without purification. The solvent acetonitrile- $d_{3}$ (99.5\% deuteration) was purchased from Deutero. Absorption of this solvent did not affect UV-Vis and IR absorption spectra in the spectral ranges investigated.

\section{Results}

In Fig. 2, the UV-absorption spectra of DPCP and DPA dissolved in acetonitrile are compared. The DPCP spectrum is dominated by the strong $S_{2} \leftarrow S_{0}$ band of $\pi \pi^{*}$ character at $260-320 \mathrm{~nm}$. The weaker $S_{1} \leftarrow S_{0}$ band at around $330 \mathrm{~nm}$ can be attributed to the $n \pi^{*}$ transition of the carbonyl group [15]. For DPA the strong absorption band at $295 \mathrm{~nm}$ is attributed to the optically allowed $S_{2} \leftarrow S_{0}\left({ }^{1} B_{1 \mathrm{u}} \leftarrow{ }^{1} A_{\mathrm{g}}\right)$ transition, although three other excited states have been identified in this energy window [16-18]. The one with the lowest energy $\left({ }^{1} A_{\mathrm{u}}\right)$ is assigned to the $S_{1}$ state and characterized by an elongated central C-C bond. The $S_{1} \leftarrow S_{0}$ transition is one-photon forbidden.

\subsection{Diphenylacetylene}

Transient IR-spectra. In a first series of experiments, transient spectra of DPA were determined, which served as a reference for later measurements on DPCP. Figure 3a shows transient IR spectra of DPA in $\mathrm{CD}_{3} \mathrm{CN}$ measured 25 and 1500 ps after populating the $S_{2}$ state with a $267 \mathrm{~nm}$ pump pulse. For comparison, the linear IR absorption spectrum of DPA is shown in Fig. 3b. In the plotted frequency range, the stationary spectrum 


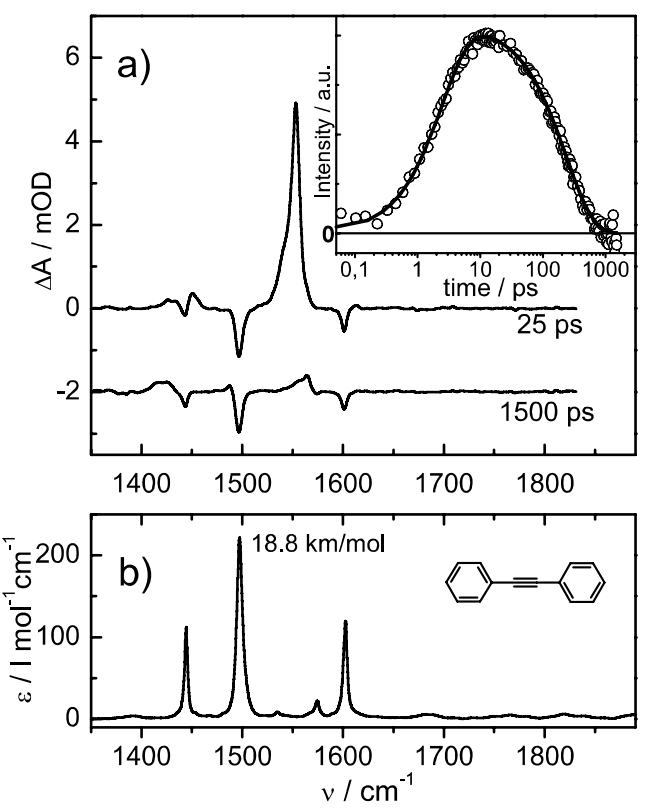

Fig. 3. IR spectra of DPA in $\mathrm{CD}_{3} \mathrm{CN}$ : (a) transient IR spectra after $267 \mathrm{~nm}$ excitation at 25 and $1500 \mathrm{ps}$ pump-probe delay (for clarity the 1500 ps spectrum is vertically shifted by $-2 \mathrm{mOD}$ ), the inset shows the spectral evolution of the $1553 \mathrm{~cm}^{-1}$ absorption band attributed to the $S_{1}$ state (open circles: experimental data, solid line: fit using Eq. 1) with $\tau_{S 2}=2.5 \pm 0.2 \mathrm{ps}$ and $\tau_{S 1}=220 \pm 20 \mathrm{ps}$ ); (b) stationary absorption spectrum.

consists of three relatively weak absorption bands originating from out of phase ring vibrations 18b, 19a, and 8a (using Wilson's notation for benzene) at 1444, 1498, and $1602 \mathrm{~cm}^{-1}$, respectively [19] The strongest peak at $1498 \mathrm{~cm}^{-1}$ has an intensity of $18.8 \mathrm{~km} / \mathrm{mol}$ and will serve as a marker band for the DPCP photolysis experiments (see below). Upon photo-excitation, these bands appear as bleaches (negative absorptions) in the transient spectrum, whereas positive peaks originate from excited states (Fig. 3a). At a pump-probe delay of $25 \mathrm{ps}$, two positive absorption bands at $1445 \mathrm{~cm}^{-1}$ (superimposed on ground state bleach) and $1553 \mathrm{~cm}^{-1}$ are observed. These lines have been identified before and were assigned to $19 \mathrm{a}$ and $8 \mathrm{a}$ modes of the DPA $S_{1}$ state, respectively [19]. A weak absorption at $1580 \mathrm{~cm}^{-1}$ at earlier times attributed to the initially populated $S_{2}$ does not emerge in our transient IR spectra [19] The $S_{1}$ precursor can be deduced only from the kinetics of the strong $1553 \mathrm{~cm}^{-1}$ band. As shown in the inset of Fig. 3a this band shows a rise followed by a slower decay (note the logarithmic time scale). On the basis of the established DPA relaxation sequence $S_{2} \rightarrow S_{1} \rightarrow T_{1}$ a function of the form

$$
f(t)=A_{1} \exp \left(-t / \tau_{S 1}\right)-A_{2} \exp \left(-t / \tau_{S 2}\right)
$$

was fitted to the data yielding $S_{2}$ and $S_{1}$ lifetimes for DPA of $\tau_{S 2}=2.5 \pm 0.2$ ps and $\tau_{S 1}=$ $220 \pm 20$ ps (full line in the inset of Fig. 3a). 


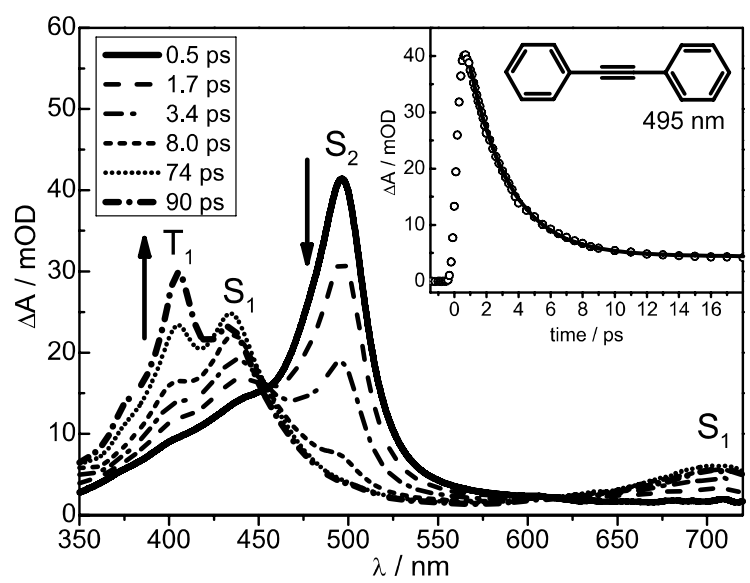

Fig. 4. Transient spectra of DPA in acetonitrile in the UV/Vis range after excitation at $260 \mathrm{~nm}$. Absorption bands attributed to $S_{2}, S_{1}$, and $T_{1}$ state are indicated. The inset shows the kinetics of the $S_{2}$ state at $495 \mathrm{~nm}$. The solid line is an exponential fit with a time constant of $\tau_{S 2}=2.5 \mathrm{ps}$.

Whereas $\tau_{S 2}$ is three times shorter than the reported value of $8-9 \mathrm{ps}$, the $S_{1}$ lifetime is in good agreement with earlier measurements $[6,7,16]$. The eventually populated $T_{1}$ state of DPA manifests itself in positive absorptions at 1425,1490 , and $1560 \mathrm{~cm}^{-1}$ remaining at a pump-probe delay of $1500 \mathrm{ps}$ (Fig. 3a).

An important feature of the transient IR spectra in Fig. 3 is that they allow for estimating absolute intensities of absorption bands in the excited states. At 25 ps delay, almost all the photo-excited DPA molecules are in the $S_{1}$ state. As the intensities of ground state bands are known, those for the excited state are easily calculated from the integrated band intensity ratio of excited state absorptions to ground state bleach. In this way, from a comparison with the ground state absorption band at $1498 \mathrm{~cm}^{-1}$ (intensity $18.8 \mathrm{~km} / \mathrm{mol}$, see Fig. 3b), an intensity of $130 \pm 20 \mathrm{~km} / \mathrm{mol}$ is obtained for the strong $S_{1}$ absorption band of DPA at $1553 \mathrm{~cm}^{-1}$. (We have to use integrated band intensities instead of extinction coeffients for a quantitative comparison here, because the ground state bands are fully resolved by FT-IR spectroscopy, whereas the $\sim 3 \mathrm{~cm}^{-1}$ resolution of our transient IR spectrometer is not sufficient to resolve the line width, which leads to a decrease of the observed peak intensity in the transient spectra.)

Transient electronic spectra. In order to validate the time constants of the excited states of DPA, we measured transient spectra in the near UV to visible spectral range. Results are shown in Fig. 4 for DPA in $\mathrm{CH}_{3} \mathrm{CN}$. Directly after $260 \mathrm{~nm}$ excitation, a strong absorption of the $S_{2}$ at $495 \mathrm{~nm}$ appears. The decay of this peak is accompanied by a rise of absorption bands at 435 and $700 \mathrm{~nm}$ attributed to the $S_{1}$ state. Subsequently, the $S_{1}$ slowly transforms into $T_{1}$ population showing up as a peak at $405 \mathrm{~nm}$. These findings completely agree with previous experiments $[6,7,16]$, however, again the $S_{2}$ lifetime we observe is considerably shorter than reported. This is shown in the inset of Fig. 4 where the exponential fit to the decay at $495 \mathrm{~nm}$ yields a time constant of $\tau_{S 2}=2.5 \pm 0.2 \mathrm{ps}$, in excellent agreement with the result of our IR experiment. 

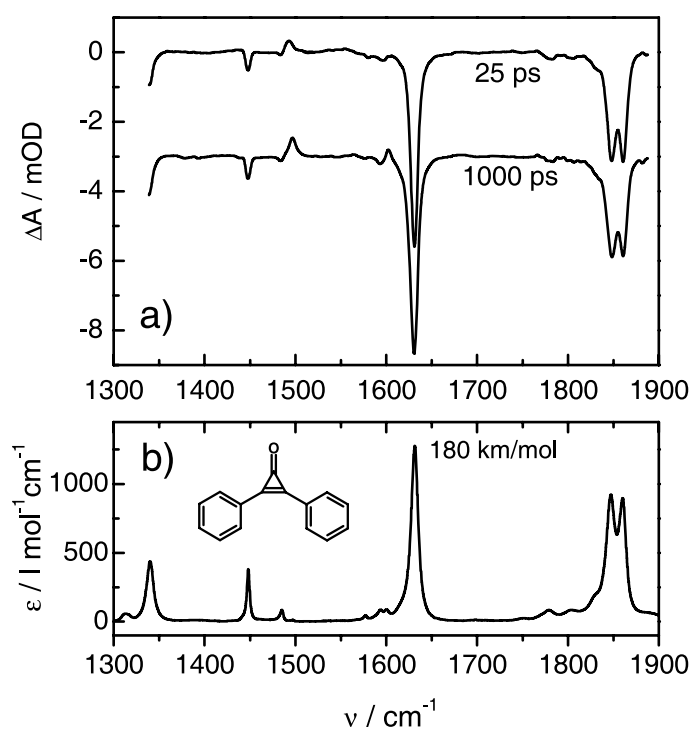

Fig. 5. IR spectra of DPCP in $\mathrm{CD}_{3} \mathrm{CN}$ : (a) transient IR spectra after $267 \mathrm{~nm}$ excitation at 25 and $1000 \mathrm{ps}$ pump-probe delay (the 1000 ps spectrum is vertically shifted by $-3 \mathrm{mOD}$ ); (b) stationary absorption spectrum.

An analysis of equivalent transient spectra obtained in the non-polar solvent $n$ hexane gives the time constants $\tau_{S 2}=5.1 \pm 0.5 \mathrm{ps}$ and $\tau_{S 1}=200 \pm 20 \mathrm{ps}$. It appears that the different values found for $\tau_{S 2}$ in acetonitrile and $n$-hexane may be attributed to variation in solvent polarity.

\subsection{Diphenylcyclopropenone}

In the following, time-resolved experiments on DPCP are presented and compared to those of DPA. Note that these experiments require always freshly prepared samples of DPCP and a flow rate in the sample cell of $>30 \mathrm{~cm} / \mathrm{s}$ to prevent accumulation of the photoproduct DPA in the region of the laser focus. Otherwise strong signals from DPA are difficult to suppress. For weak signals requiring long data acquisition times this turned out to be rather problematic.

Transient IR spectra. Figure 5a shows IR spectra measured 25 and $1000 \mathrm{ps}$ after $267 \mathrm{~nm}$ excitation, respectively. They mainly consist of negative peaks due to the bleached ground state of DPCP, as is evident from a comparison with the stationary spectrum shown in Fig. 5b. The strong absorptions at 1632 and $1854 \mathrm{~cm}^{-1}$ can be assigned to in-phase and out-of-phase combination vibrations of the central double bond and the $\mathrm{C}-\mathrm{O}$ stretch, respectively. Together with less IR active ring vibrations at 1340, 1448 , and $1485 \mathrm{~cm}^{-1}$ these bands appear as direct mirror images in the transient spectra. Additionally, positive peaks appear in Fig. 5a indicating the formation of a photoproduct. At $25 \mathrm{ps}$ time delay, only a single absorption at $1498 \mathrm{~cm}^{-1}$ is immediately visible, coinciding with the corresponding band of DPA in the ground electronic state $(c f$. the 
stationary spectrum of Fig. 3a). The two other lines of ground state DPA in the frequency window considered are covered by DPCP bleach $\left(1448 \mathrm{~cm}^{-1}\right)$ or are visible only in the 1000 ps spectrum $\left(1602 \mathrm{~cm}^{-1}\right)$. The latter can be explained by the fact that DPA is formed vibrationally hot in the ground state, which initially leads to a broadening of the lines. As the cooling process takes several tens of picoseconds, the bands sharpen, such that the $1602 \mathrm{~cm}^{-1}$ band becomes visible.

We were not able to observe the formation of carbon monoxide at $2130 \mathrm{~cm}^{-1}$. The reason could be that immediately after photodissociation the $\mathrm{CO}$ molecule is vibrationally highly excited and our time window is too short to observe the relaxation. Consequently, the corresponding IR band would be too broad to be distinguishable from an overall offset of the spectrum.

The stationary spectra of Figs. $3 \mathrm{~b}$ and $5 \mathrm{~b}$ show that the intensities of the strongest bands of DPA $\left(1498 \mathrm{~cm}^{-1}, 18.8 \mathrm{~km} / \mathrm{mol}\right)$ and of DPCP $\left(1632 \mathrm{~cm}^{-1}, 180 \mathrm{~km} / \mathrm{mol}\right)$ differ by a factor of $\sim 10$. The amplitude ratio of these bands (bleach for DPCP and absorption for DPA) in the transient spectrum at 1000 ps (Fig. 5a) has the same value, indicating a quantum yield of 1.0 for DPA in the $S_{0}$ state. If DPA was produced in an excited electronic state, we should observe absorption from the $S_{1}$ state at $1553 \mathrm{~cm}^{-1}$ after 25 ps similar to Fig. 3a. Furthermore, this band should have similar amplitude as the strong DPCP bleach at $1632 \mathrm{~cm}^{-1}$ since both lines are almost equally intense. Instead at the expected position at $1553 \mathrm{~cm}^{-1}$, only a slight elevation is seen, indicating $<3 \%$ of DPA in the $S_{1}$ state. Such a small amount can be explained by an impurity of DPA accumulated in the experiment as we will show below.

This conjecture is further supported by transient IR spectra measured with variable pump wavelength in the range $267-340 \mathrm{~nm}$ (Fig. 6, all taken at $25 \mathrm{ps}$ pump-probe delay). For clarity, these spectra are normalized to the bleached $1632 \mathrm{~cm}^{-1}$ mode of ground state DPCP and vertically shifted. The pump wavelength of each spectrum is denoted at the ordinate.

For long wavelength excitation, i.e. $\lambda_{\text {pump }}>310 \mathrm{~nm}$, no absorption from the DPA $S_{1}$ state at $1553 \mathrm{~cm}^{-1}$ can be observed. Only at higher excitation energy a small peak from the excited state emerges. Comparison with the UV absorption spectrum in Fig. 2 reveals that the transition appears just at the onset of the $S_{2} \leftarrow S_{0}$ absorption of DPA, suggesting direct excitation of DPA impurities at $\lambda_{\text {pump }}<310 \mathrm{~nm}$. In contrast, at longer pump wavelength only photo-dissociation of DPCP is possible whereas a DPA contamination cannot be excited. Another important feature which can be inferred from Fig. 6 is that there seems to be no dependence of the outcome of the reaction on whether the $S_{1}$ or the $S_{2}$ state of DPCP is initially excited. The spectra with $330\left(S_{1}\right)$ and $315 \mathrm{~nm}\left(S_{2}\right)$ excitation wavelength are almost identical.

Transient electronic spectra. Since the transient IR spectra did not show any signature of the initially excited state of DPCP, its dynamics were investigated in the UV/Vis region. The data presented in Fig. 7 show that the decay of the DPCP $S_{2}$ state is close to the experimental time resolution. Therefore, the transient spectra of Fig. 7 were carefully selected at time delays with no significant overlap of pump and probe pulses. The upper panel shows a broad DPCP excited state absorption extending from beyond 350 to $530 \mathrm{~nm}$ with a peak centred at $470 \mathrm{~nm}$. The decay time of $230 \pm 30 \mathrm{fs}$ is in excellent agreement with previous measurements [7]. At $1.3 \mathrm{ps}$ an offset spectrum remains 


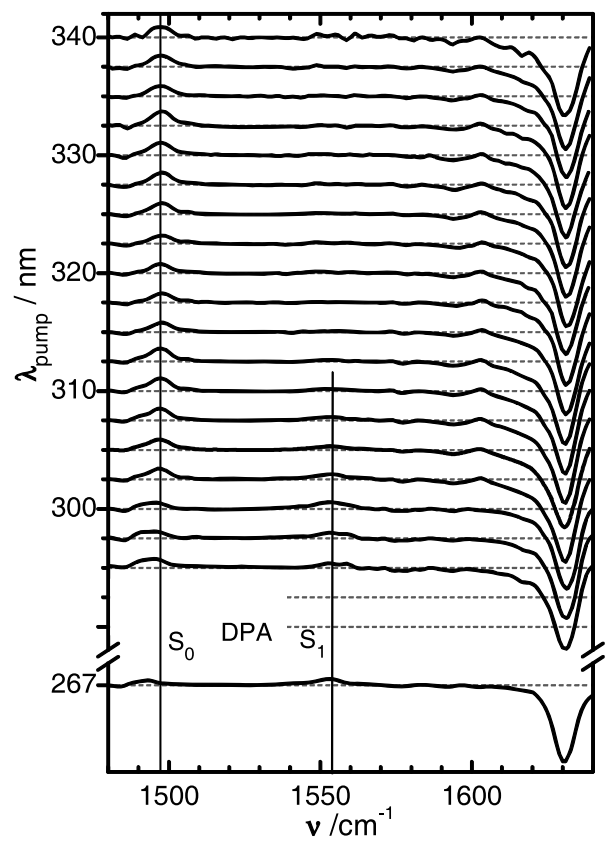

Fig. 6. Dependence of transient IR spectra of DPCP on the pump wavelength (solvent: $\mathrm{CD}_{3} \mathrm{CN}$ ). The spectra were measure at constant pump-probe delay of $25 \mathrm{ps}$, scaled to equal bleaching signal of ground state DPCP at $1632 \mathrm{~cm}^{-1}$, and vertically shifted. The ordinate denotes the pump wavelength.

which we attribute to a superposition of DPA in the $S_{2}$ state with a peak at $500 \mathrm{~nm}$ and of a DPCP ground state bleach at $360 \mathrm{~nm}$. (Note that the $S_{1} \leftarrow S_{0}$ band of DPCP in $n$ hexane is red-shifted considerably with respect to that in acetonitrile solution and peaks at $360 \mathrm{~nm}$ [7].)

Based on our analysis of the transient IR spectra, we attribute the $S_{2}$ absorption of DPA in Fig. 7 to direct excitation of a DPA impurity. We may estimate its contribution Since the $S_{2}$-absorption bands of DPA in Figs. 4 and 7 were obtained under almost identical experimental conditions (pump intensity, optical density), a comparison of their intensities allows us to estimate a contamination of $<5 \%$. A comparison of the 1.3 and 1.8 ps transients of Fig. 7 indicates that there is essentially no temporal evolution of the offset spectrum over a period of $0.5 \mathrm{ps}$. This enables the calculation of the bare excited state spectrum of DPCP from the difference $\Delta A(\lambda, t)-\Delta A(\lambda, 1.8 \mathrm{ps})$. The outcome is presented in the lower panel of Fig. 7 clearly showing $S_{2}$ absorption of DPCP in the near $\mathrm{UV}$, a result which is in marked contrast to previous measurements [7].

Origin of excited DPA in the $S_{2}$-state. The observed most prominent $S_{1}$-state absorption of DPA at $1553 \mathrm{~cm}^{-1}$ is by far smaller than to be expected from the DPCP carbonyl bleach. Likewise, the near UV absorption of the DPA $S_{2}$-state occurring in photolysis experiments of DPCP is much smaller than to be expected. This indicates that direct excitation of ground state DPA present in the sample is responsible for these observations, for which we suggest three possible sources: 


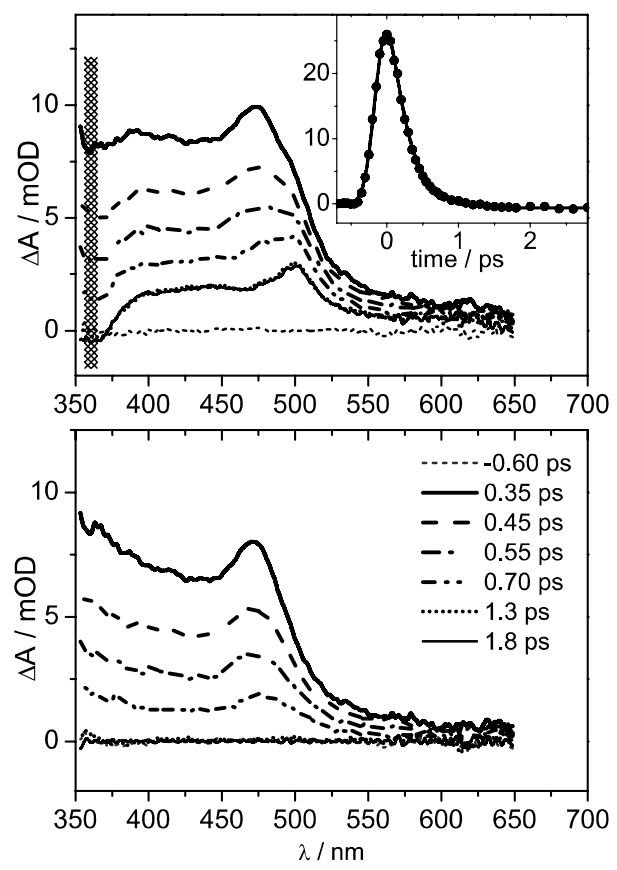

Fig. 7. Upper panel: transient UV/Vis-spectra of DPCP in acetonitrile after excitation at $260 \mathrm{~nm}$ for pumpprobe delays as indicated; the inset shows the decay at $360 \mathrm{~nm}$ (shaded region), the solid line is a fit to the data points based on a convolution of a Gaussian-shaped instrument response function of $300 \mathrm{fs}$ FWHM and an exponential decay with a time constant of 230 fs. Lower panel: Excited state spectra of DPCP constructed from the difference to the offset spectrum at $1.8 \mathrm{ps}$ in the upper panel.

1. It is already present from the start,

2. it accumulates during the measurement because of too slow flow rate, and/or

3. it is formed during the pump pulse following decarbonylation of DPCP and excited by its trailing edge.

(i) As high purity DPCP with less than $0.05 \%$ DPA was employed, the first source is of negligible importance. (ii) As for DPA accumulation, the sample flow rate was $>30 \mathrm{~cm} / \mathrm{s}$, ensuring replacement of the excitation volume between successive laser shots. Its effect can be minimized by applying short data acquisition times. (iii) DPA excitation by the trailing edge of the pump pulse can be modelled using the kinetic scheme

$$
\begin{aligned}
& \operatorname{DPCP}\left(S_{0}\right) \stackrel{k_{1}(t)}{\longrightarrow} \operatorname{DPCP}\left(S_{2}\right) \\
& \operatorname{DPCP}\left(S_{2}\right) \stackrel{k_{2}}{\longrightarrow} \operatorname{DPA}\left(S_{0}\right)+\mathrm{CO} \\
& \operatorname{DPA}\left(S_{0}\right) \stackrel{k_{3}(t)}{\longrightarrow} \operatorname{DPA}\left(S_{2}\right)
\end{aligned}
$$

with

$$
\begin{aligned}
& k_{1}(t)=\ln (10) \varepsilon_{\mathrm{DPCP}} I(t) \\
& k_{3}(t)=\ln (10) \varepsilon_{\mathrm{DPA}} I(t)
\end{aligned}
$$




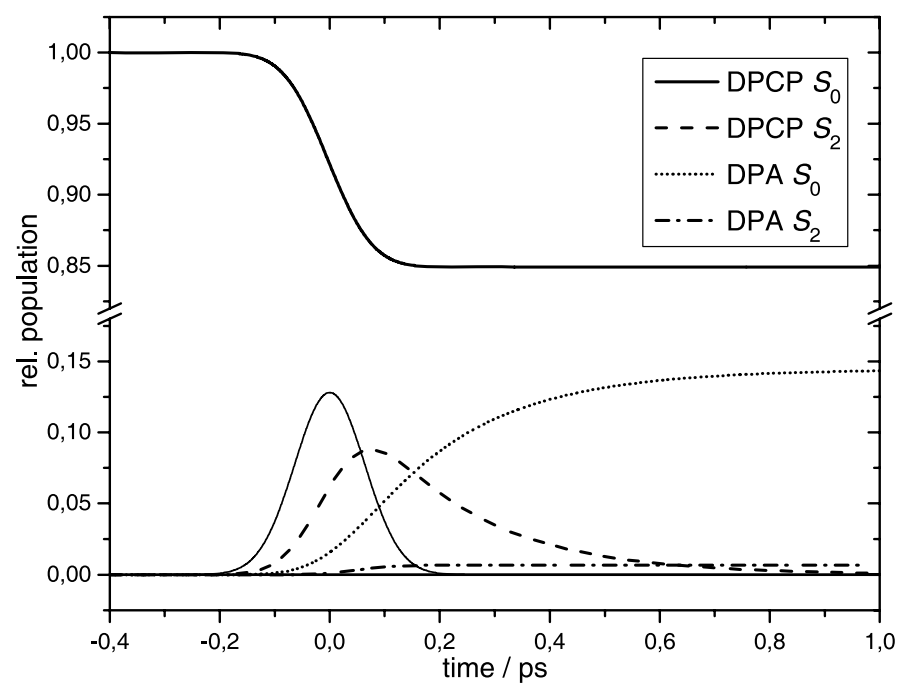

Fig. 8. Numerical calculation of population changes applying the kinetic model of Eq. (2). The excitation pulse (150 fs, $1 \mu \mathrm{J}, 200 \mu \mathrm{m}$ focus diameter) is shown by the thin line.

The time dependent excitation rate coefficients $k_{1}$ for DPCP and $k_{3}$ for DPA (Eq. 3) contain the intensity profile $I(t)$ of the pump pulse and the respective ground state molar decadic extinction coefficients at the pump wavelength of $267 \mathrm{~nm}$ for DPCP and DPA, $\varepsilon_{\mathrm{DPCP}}=10^{4} 1 \mathrm{~mol}^{-1} \mathrm{~cm}^{-1}$ and $\varepsilon_{\mathrm{DPA}}=2 \times 10^{4} 1 \mathrm{~mol}^{-1} \mathrm{~cm}^{-1}$. Decay of $\operatorname{DPCP}\left(S_{2}\right)$ and thus formation of $\operatorname{DPA}\left(S_{0}\right)$ are described by the rate constant $k_{2}=(0.2 \mathrm{ps})^{-1}$ and $\operatorname{DPA}\left(S_{0}\right)$ is subsequently excited by the same pump pulse. The kinetic model was solved numerically assuming a $150 \mathrm{fs}$ FWHM Gaussian-shaped pump pulse with $1 \mu \mathrm{J}$ energy focused down to a diameter of $200 \mu \mathrm{m}$. For simplicity, we ignore the fact that DPA is formed vibrationally hot, which could alter the extinction coefficient with respect to the room temperature value. With these assumptions the model calculation presented in Fig. 8 shows that $15.1 \%$ of ground state DPCP ( 0.849 amplitude after $1 \mathrm{ps})$ is bleached producing DPA in the ground state, of which $95.4 \%$ remain in $S_{0}$ (amplitude 0.144) while $4.6 \%$ (amplitude 0.007 ) are excited to the $S_{2}$-state by the trailing edge of the pump pulse. These relative populations are in very good agreement with our experimental results and suggest that under our experimental conditions this route represents the main source of $\operatorname{DPA}\left(S_{2}\right)$.

\section{Discussion}

From our analysis of the transient spectra presented above, there is no evidence for an adiabatic reaction channel from the $S_{2}$-state of DPCP to the $S_{2}$-state of DPA (Fig. 7). Photo-excitation of DPCP yields DPA exclusively in the ground state (Fig. 5) with unity quantum yield, such that decarbonylation pathway I (Fig. 1) has to be ruled out definitely. 


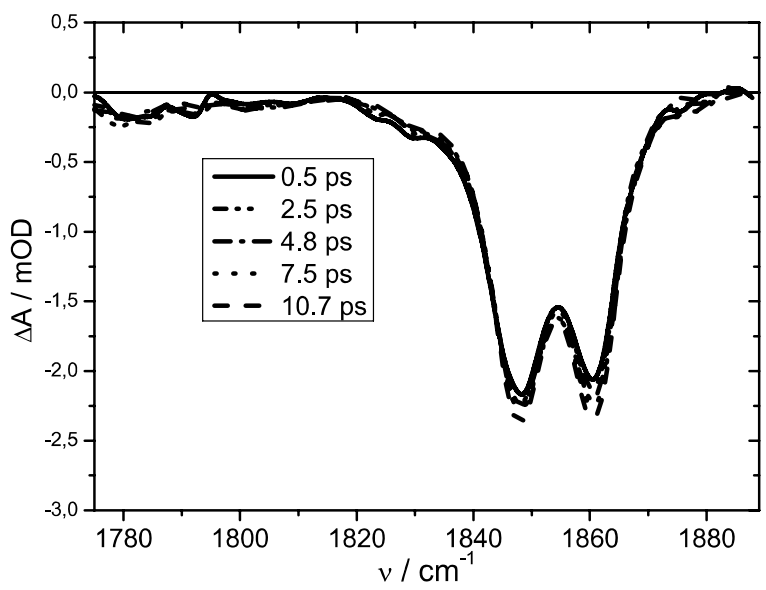

Fig. 9. Transient IR spectra of DPCP in $\mathrm{CD}_{3} \mathrm{CN}$ after excitation at $267 \mathrm{~nm}$ at selected pump-probe delays. The photo-bleached carbonyl stretch mode is not refilled by internal conversion.

We turn now to pathway II (Fig. 1) as a possible route for decarbonylation with internal conversion to ground state DPCP as the first step. After internal conversion with a time constant of $0.2 \mathrm{ps}$, the initially vibrationally hot ground state DPCP would be characterized by broadened and red-shifted IR lines (see e.g. Refs. [11-13] for the IR signature of vibrationally excited molecules). As a result, the bleach signals at 1632 and $1854 \mathrm{~cm}^{-1}$ in the difference spectrum of DPCP (Fig. 5a) would be partially filled up on a timescale of 0.23 ps. Additionally, a red-shifted absorption due to anharmonic coupling to excited low-frequency modes of the molecules should appear. However, taking a closer look at the time evolution of the prominent bleached IR DPCP ground state band at $1854 \mathrm{~cm}^{-1}$ in Fig. 9, there is no indication for a repopulation of the DPCP $S_{0}$-state.

This observation is in agreement with the transient UV/Vis spectra of Fig. 7, upper panel. There, the ground state bleach of DPCP at $360 \mathrm{~nm}$ does not change during the lifetime of the DPCP $S_{2}$-state. Our spectral evidence, therefore, strongly suggests that the ultrafast decay of the DPCP $S_{2}$-state is not associated with $S_{2} \rightarrow S_{0}$ internal conversion.

This conclusion presumes, however, that the lifetime of the hot $S_{0}$-state is longer than the $S_{2}$-lifetime of $200 \mathrm{fs}$ or, conversely, it would be invalid if decarbonylation from the hot ground state would be faster. In order to estimate a rough upper limit for the corresponding unimolecular photodissociation rate coefficient of DPCP in the $S_{0}$-state, we performed a standard RRKM-calculation assuming that the entire initial pump photon energy $E_{\text {exc }}=37450 \mathrm{~cm}^{-1}$ (wavelength $267 \mathrm{~nm}$ ) is available for the reaction in the $S_{0}$-state and that it is microcanonically distributed. Because of the large photon energy, one may neglect the initial thermal energy distribution $(T=300 \mathrm{~K})$ in the $S_{0}$-state. With these assumptions, we can calculate the specific (microcanonical) reaction rate coefficient $k\left(E_{\text {exc }}\right)$ for $\operatorname{DPCP}\left(S_{0}^{\dagger}\right) \rightarrow \operatorname{DPA}\left(S_{0}\right)$ [20] using normal mode frequencies obtained from DFT-calculations (see Appendix), a reactive mode of $1625 \mathrm{~cm}^{-1}$, and the decarbonylation energy barrier $E_{0}=11160 \mathrm{~cm}^{-1}$ calculated by Poloukhtine and Popik [10] 


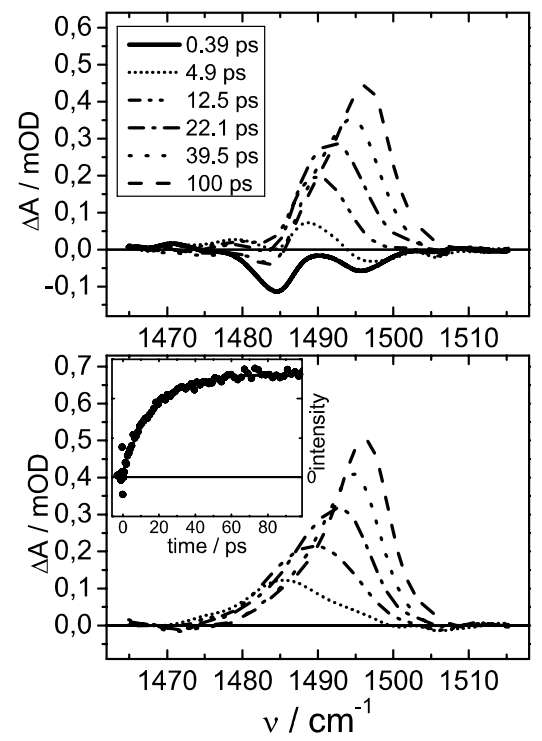

Fig. 10. Upper panel: formation of the $1498 \mathrm{~cm}^{-1}$ marker band of DPA after $267 \mathrm{~nm}$ photolysis of DPCP in $\mathrm{CD}_{3} \mathrm{CN}$ at selected pump-probe delays. Lower panel: difference spectra $\Delta A(\lambda, t)-\Delta A(\lambda, 0.39 \mathrm{ps})$ calculated from the upper panel; the inset shows the evolution of the integrated band intensity, the solid line is an exponential fit to the data points with a time constant of $16.0 \pm 1.5 \mathrm{ps}$.

We obtain a lifetime of $1 / k\left(E_{\text {exc }}\right)=20 \mathrm{~ns}$, which means that a "statistical" reaction $\operatorname{DPCP}\left(S_{0}^{\dagger}\right) \rightarrow \operatorname{DPA}\left(S_{0}\right)$ out of the hot ground state would be far too slow to compete with vibrational relaxation in solution, even if one considers a maximum factor of 10 uncertainty because of the approximations made. As an ultrafast non-statistical reaction after $S_{2} \rightarrow S_{0}$ internal conversion is most unlikely, this is a strong argument against decarbonylation pathway II (Fig. 1). As a consequence, in view of our experimental results and calculations, we are left with decarbonylation pathway III, which would be in agreement with conclusions deduced from experiments on DPCP derivatives and naphthyl substituted cyclopropenones by Poloukhtine and Popik [10]

Another experimental indication for pathway III is presented in Fig. 10 which shows the time evolution of the $1498 \mathrm{~cm}^{-1}$ marker band of DPA after excitation of DPCP (see Fig. 5a). The earliest spectrum (upper panel) at a pump-probe delay of $0.39 \mathrm{ps}$ is characterized by two negative peaks. The stronger one at $1485 \mathrm{~cm}^{-1}$ corresponds to bleached DPCP (cf. the stationary spectrum Fig. 5b), the weaker one at $1495 \mathrm{~cm}^{-1}$ can be attributed to a DPA impurity, which is bleached as well (from the intensity ratio $8 \%$ of DPA can be estimated). On the timescale of the experiment (100 ps) both bleaches are permanent. Therefore, the 0.39 ps spectrum can be subtracted to obtain the time evolution of the undistorted band of newly formed DPA which is plotted accordingly in the lower panel of Fig. 10. It clearly shows the signature of vibrationally hot DPA molecules cooling down within several tens of ps. In many cases the IR band intensity of a large molecule is independent on the vibrational excitation such that the band integral can be used as a measure of the population. For the photo-generated DPA 
molecule this does not seem to be the case as the band integral builds up with a time constant of $16.0 \pm 1.5 \mathrm{ps}$ (see inset in the lower panel of Fig. 10). Since the excited state of DPCP has a lifetime of only 0.23 ps we expect to observe the formation of DPA with the same time constant. The discrepancy can be explained by the fact that the $1498 \mathrm{~cm}^{-1}$ mode involves out of phase ring vibrations of $\mathrm{C}-\mathrm{H}$ bend character delocalised over both phenyl groups. If DPA is vibrationally hot, low frequency ring torsions are excited which distort the co-planar geometry as well as the coupling between both rings. As a consequence, the intensity of the IR transition might be reduced.

\section{Conclusion}

To elucidate the photodecarbonylation of diphenylcyclopropenone (DPCP), we conducted UV/Vis and IR femtosecond pump-probe experiments on both diphenylacetylene (DPA) and DPCP. A reinvestigation of DPA photophysics largely confirmed the results of previous studies $[6,7,17]$. With our better experimental time resolution, we obtained lifetimes of the initially excited DPA $S_{2}$ state that are shorter than reported previously and show a significant solvent polarity dependence, i.e. $2.5 \pm 0.2 \mathrm{ps}$ in acetonitrile and $5.1 \pm 0.5 \mathrm{ps}$ in $n$-hexane. The lifetimes of the subsequently populated DPA $S_{1}$-state of $220 \pm 20 \mathrm{ps}$ in acetonitrile and $200 \pm 20 \mathrm{ps}$ in $n$-hexane match well with those reported by others. Moreover, the absolute intensity of DPA $S_{1}$-state absorption at $1553 \mathrm{~cm}^{-1}$ of $130 \pm 20 \mathrm{~km} / \mathrm{mol}$ was obtained by comparison with the $S_{0}$ state stationary spectrum.

For DPCP photodecarbonylation we discussed the three possible reaction pathways suggested in the literature $[6,7,9,10,16]$ :

1. Excited state surface adiabatic dissociation to the DPA $S_{2}$-state.

2. Internal conversion and reaction from a hot electronic ground state.

3. Non-adiabatic dissociation from the DPCP $S_{2}$-state to the DPA $S_{0}$-state.

The adiabatic path $\mathrm{I}$ is to be dismissed based on three grounds:

1. The observed most prominent $S_{1}$-state absorption of DPA at $1553 \mathrm{~cm}^{-1}$ is by far smaller than to be expected from the DPCP carbonyl bleach. Likewise, the near UV absorption of the DPA $S_{2}$-state occurring in photolysis experiments of DPCP is much smaller than to be expected. To explain the observation of excited states of DPA, a model describing the excitation of DPA formed by the trailing edge of the pump pulse was employed and succeeds in explaining the small absorptions observed.

2. Experiments with pump wavelengths covering the edges of the respective $S_{2}$-state absorptions of DPA and DPCP clearly showed that the DPA $S_{1}$-state IR absorption band appears only due to direct excitation of DPA contamination and that the IR transient absorption spectrum generated by DPCP excitation essentially is the same for DPCP $S_{2}$ - and $S_{1}$-state excitation.

3. Hot ground state DPA was observed at the $1498 \mathrm{~cm}^{-1}$ marker band immediately after the pump pulse. The intensity of the absorption reaches its final plateau value not until after a few tens of picoseconds which can be explained by decreased oscillator strengths as a result of vibrational excitation. 
The hot ground state reaction pathway II can be ruled out as hot ground state absorptions of DPCP were not observed in transient IR spectra at short time delays and calculations show that a reaction from the vibrationally excited $S_{0}$-state would be orders of magnitude too slow to account for DPA formation.

Therefore it appears that pathway III is the route by which decarbonylation takes place, i.e. a non-adiabatic process starting in the DPCP $S_{2}$-state and leading directly to DPA in its $S_{0}$-state. Poloukhtine and Popik [10] suggested a similar reaction path for the corresponding decarbonylation of DPCP derivatives and naphthyl substituted cyclopropenones on the basis of transient electronic spectra. For dianisylcyclopropenone (ACPC), e.g., they observed an intermediate with a rise time of $350 \mathrm{fs}$ and a decay time of $624 \mathrm{fs}$ at $454 \mathrm{~nm}$ in methanol. Based on DFT-calculations they assigned this absorption to an intermediate state along the non-adiabatic reaction path from electronically excited ACPC to ground state dianisylacetylene. We did not find any evidence for such an intermediate in the decarbonylation of DPCP, so the question remains open, whether DPCP is a special case or the electronic assignment in Ref. [10] should be revised. Femtosecond broadband transient IR spectroscopy could help settle this point.

\section{Appendix}

Normal mode frequencies of DPCP in the $S_{0}$-state required to estimate the specific rate constant $k\left(E_{\text {exc }}\right)$ by RRKM-theory were obtained from DFT-calculations (BP86TZVPP) using the ORCA-package [21-23] and subsequently scaled by a correction factor of 1.004 .

Table 1. Scaled normal mode frequencies of DPCP $\left(S_{0}\right)$ in $\mathrm{cm}^{-1}$.

313631363128312631223121311431123107310618641625159615901567156514861472 144114401339133513131309129311751169115911581123108310781033102610111001 996987984977971952919911833830772765749718699693689654614611608516468 42841139837327024123514612894765741

\section{Acknowledgement}

Financial support by the Deutsche Forschungsgemeinschaft is gratefully acknowleged.

\section{References}

1. G. Quinkert, K. Opitz, W. W. Wiersdorff, and J. Weilich, Tetrahedron Lett. 4 (1963) 1863.

2. R. W. Fessenden, P. M. Carton, H. Shimamori, and J. C. Scalano, J. Phys. Chem. 86 (1982) 3803.

3. T. Hara, N. Hirota, and M. Terazima, J. Phys. Chem. 100 (1996) 10194-10200.

4. R. R. Hung and J. J. Grabowski, J. Am. Chem. Soc. 114 (1992) 351-353.

5. M. S. Herman and J. L. Goodman, J. Am. Chem. Soc. 111 (1989) 1849-1854.

6. Y. Hirata and N. Mataga, Chem. Phys. Lett. 193 (1992) 287-291.

7. S. Takeuchi and T. Tahara, J. Chem. Phys. 120 (2004) 4768-4776. 
8. G. Kuzmanich, A. Natarajan, K. K. Chin, M. Veerman, C. J. Mortko, and M. A. GarciaGaribay, J. Am. Chem. Soc. 130 (2008) 1140-1141.

9. L. T. Nguyen, F. de Proft, M. T. Nguyen, and P. Geerlings, J. Chem. Soc. Perkin Trans. 2 (2001) 898-905.

10. A. Poloukhtine and V. Popik, J. Phys. Chem. A 110 (2006) 1749-1757.

11. C. Reichardt, J. Schroeder, and D. Schwarzer, Phys. Chem. Chem. Phys. 10 (2008) 5218.

12. C. Reichardt, J. Schroeder, P. Vöhringer, and D. Schwarzer, Phys. Chem. Chem. Phys. 10 (2008) 1662-1668.

13. C. Reichardt, J. Schroeder, and D. Schwarzer, J. Phys. Chem. A 111 (2007) 10111-10118.

14. R. A. Kaindl, M. Wurm, K. Reimann, P. Hamm, A. M. Weiner, and M. Woerner, J. Opt. Soc. Am. B 17 (2000) 2086-2094.

15. H. Yishida and H. Miyahara, Bull. Chem. Soc. Jpn. 45 (1972) 1919.

16. Y. Hirata, T. Okada, N. Mataga, and T. Nomoto, J. Phys. Chem. 96 (1992) 6559-6563.

17. C. Ferrante, U. Kensy, and B. Dick, J. Phys. Chem. 97 (1993) 13457-13463.

18. K. Okuyama, T. Hasegawa, M. Ito, and N. Mikami, J. Phys. Chem. 88 (1984) 1711.

19. T. Ishibashi, H. Okamoto, and H. Hamaguchi, Chem. Phys. Lett. 325 (2000) 212-218.

20. J. Troe, in: Physical Chemistry. An Advanced Treatise, H. Eyring, D. Henderson, and W. Jost (Eds.), Vol VIB, Kinetics of Gas Reactions, Academic Press, New York, San Francisco, London 1975.

21. F. Neese, ORCA - An ab initio, DFT and semiempirical SCF-MO package, Version 2.6.00 University of Bonn, 2007.

22. F. Neese, J. Comput. Chem. 24 (2003) 1740.

23. F. Neese, J. Am. Chem. Soc. 128 (2006) 10213. 